\title{
Options for Intraoperative Repair of a Cut Pilot Balloon on the Endotracheal Tube
}

\author{
Kwaku Owusu-Bediako' \\ Henry Turner III' \\ Omar Syed' \\ Joseph Tobias (iD) ${ }^{1,2}$ \\ 'Department of Anesthesiology \& Pain \\ Medicine, Nationwide Children's \\ Hospital, Columbus, OH, USA; \\ ${ }^{2}$ Department of Anesthesiology \& Pain \\ Medicine, The Ohio State University \\ College of Medicine, Columbus, \\ $\mathrm{OH}$, USA
}

\begin{abstract}
Severing of the pilot balloon of an endotracheal tube (ETT) results in cuff deflation and may lead to complications including inadequate patient ventilation, increased risk of aspiration and infection, and operating room air pollution with anesthetic gases. In situations where ETT exchange or reintubation may pose a significant risk to the patient, temporary repair of the severed cuff tubing can be helpful until it is safe to address the problem with replacing the ETT. Simple and effective repair methods can be achieved using readily available materials in the operating room, including intravenous cannulas, hypodermic syringes, and epidural clamp connectors. However, choosing which technique or method depends mainly on personal preference, equipment availability, and provider comfort and experience. We present a 12-year-old adolescent who presented for anesthetic care for extensive burn injury. During removal of the dressing around the head and face, the tubing of the pilot balloon of the ETT was inadvertently cut. Options for dealing with such problems are discussed, including techniques to allow for temporary repair and re-inflation of the deflated cuff.
\end{abstract}

Keywords: endotracheal tube, pilot balloon, pediatric airway

\section{Introduction}

Endotracheal tubes (ETTs) are essential for the safe conduct of anesthesia and airway management of the critically ill patient. ETTs facilitate the safe delivery of anesthetic gases, protection of the airway, positive pressure ventilation, pulmonary toileting, and maintenance of oxygenation. The use of cuffed ETTs in all pediatric age groups, especially those aged 8 years or younger, has only gained prominence in pediatric anesthesia practice in recent years. ${ }^{1}$ Cuffed ETTs may provide several advantages over uncuffed ETTs, including improved sealing of the airway, limited gas leak with improved ventilation and oxygenation, decreased need for ETT exchange, and reduced risk of aspiration. ${ }^{2-5}$ Furthermore, the effective sealing of the airway provided by the cuffed ETT helps prevent oropharyngeal contamination with anesthetic gases and oxygen, thereby limiting the risk of airway fire. ${ }^{2,6}$

The cuffed ETT is equipped with multiple design features that ensure its efficiency and safety. One such feature is the pilot balloon and valve. The pilot balloon is fitted to a spring-loaded valve with a bulb connecter attached, which allows for a rough tactile and visual confirmation of cuff inflation after endotracheal intubation or deflation before extubation. However, damage to the pilot balloon, the tubing, and valve may occur inadvertently during manipulation of the ETT or surgical procedures near the airway or face. This can result in cuff deflation, a reduction in intracuff pressure with ineffective sealing of the airway, and air leak. In addition, cuff deflation may lead to
Correspondence: Kwaku Owusu-Bediako Department of Anesthesiology \& Pain Medicine, Nationwide Children's Hospital, 700 Children's Drive, Columbus, $\mathrm{OH}$ 43205, USA

Tel $+1614722-4200$

Fax $+1614722-4203$

Email Kwaku.Owusu-

Bediako@nationwidechildrens.org 
inadequate ventilation, risk of aspiration of oropharyngeal secretions, unreliable monitoring of ventilator parameters, and the potential for airway fire when higher concentrations of oxygen are used. ${ }^{1}$ The need to use high gas flows in the event of an air leak may result in unnecessary exposure of the operating room staff to volatile anesthetic agents and have a significant economic impact, especially in low-resource settings. ${ }^{4}$ We present a 12-year-old adolescent who was receiving intraoperative anesthetic care during care for a burn injury. During removal of the dressing around the head and face, the pilot balloon inflation tubing for the ETT was inadvertently cut. Options for dealing with such problems are discussed, including techniques to allow for temporary repair and re-inflation of the deflated cuff.

\section{Case Report}

Review and presentation of this case were in accordance with the Institutional Review Board at Nationwide Children's Hospital (Columbus, Ohio). The informed consent form at Nationwide Children's Hospital allows for deidentifiable information related to a patient's anesthetic care to be used for teaching and publication. Written informed consent for anesthetic care and publication was obtained from a parent prior to the procedure. A 12-yearold, $48.3 \mathrm{~kg}$ adolescent male with a past medical history of ADHD, generalized anxiety disorder, and behavioral issues, presented for debridement and dressing of partialthickness burns of the trunk, bilateral extremities, and face (20-29\% TBSA) with split thickness skin graft and placement of skin substrate on the face. He had been in the intensive care unit (ICU) receiving mechanical ventilation for 3 weeks prior to the procedure, with burn injury and a hospital course complicated by inhalational injury with respiratory failure, hypoxic encephalopathy, and wound infection. He was premedicated with midazolam (4mg) and transported to the OR, where standard American Society of Anesthesiologists' monitors were placed. Anesthesia was induced with propofol and maintained with sevoflurane, dexmedetomidine, and hydromorphone. Anesthetic care was uneventful until shortly before the end of the procedure when during the change of wound dressing on the face, the pilot balloon for the size 5.5 endotracheal tube in situ was inadvertently cut off (Figure 1). Immediately following this, a hemostat was placed on the remainder of the pilot cuff tubing to secure the remaining air and maintain the pressure in the cuff. Ventilation was also maintained with increased flows to compensate for air leaks. The patient remained stable, and once the dressing

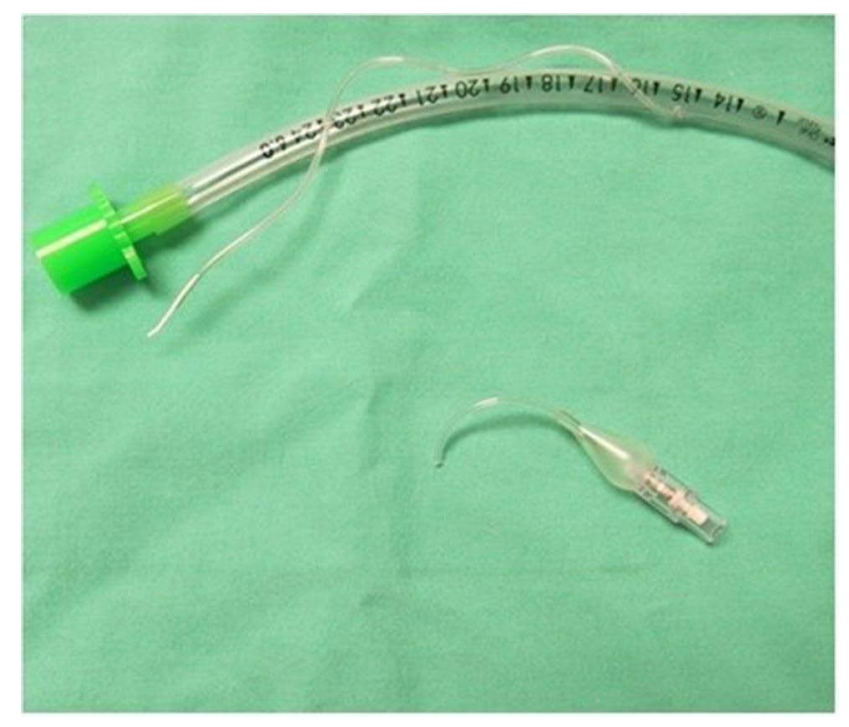

Figure I Severed pilot balloon, separated from the tubing of a cuffed endotracheal tube.

was secure, his trachea was re-intubated. This event led to a departmental quality improvement investigation with a literature search and development of an in vitro technique to determine an effective means to re-inflate the cuff of an endotracheal tube in the event that immediate exchange was not feasible.

\section{Discussion}

Centuries of innovations have led to the development of a new generation of endotracheal tubes. The Greek philosopher Hippocrates first described endotracheal intubation to support ventilation in humans $\left(460-380\right.$ BCE) ${ }^{7}$ However, it was not until the 16th century when Andreas Vesalius, an anatomist, and physician first described the rhythmic inflation of the lungs of a pig by using a reed placed into the trachea to facilitate ventilation. ${ }^{7,8}$ Years of trial and error and innovations by various physicians and investigators improved different designs and versions of the endotracheal tube and led to the development of the flexible modern ETT that allows for the safe passage through the vocal cords. Several modifications to the ETT improve its performance, including Murphy's eye, beveled tip, radio-opaque line, and an intubating stylet.

It was widely accepted that the larynx in infants and children was funnel or conical shape with the narrowest point at the cricoid ring. ${ }^{2}$ This tenet was based mainly on descriptions of the pediatric larynx by Dr Eckenhoff and studies by Bayeux in 1897, who used plaster castings and anatomical sections of cadaveric tracheas of children., 
Their conclusions led to the widespread use of uncuffed ETTs in infants and children, especially those less than 8 years old. However, recent studies using high-resolution diagnostic imaging modalities have yielded contrasting findings. Litman et al using MR imaging and Dalal et al using direct video bronchoscopic measurements of the pediatric airway, both concluded that unlike the historical findings of Bayeux and Eckenhoff, the narrowest portion of the pediatric larynx is the transverse diameter at the subglottic region, which is also relatively distensible, while the cricoid remains an area of fixed resistance to the passage of an ETT. ${ }^{10,11}$ They also reported that the anteroposterior diameter of the pediatric larynx is cylindrical rather than funnel-shaped, and therefore did not exhibit an agebased transition to the more cylindrical adult airway. ${ }^{2,12}$ These conclusions have led to the development, and increased use of the new generation cuffed ETTs in infants and children.

Proper handling and manipulation of the ETTs are needed to prevent accidental damage to or tearing of the pilot balloon and its tubing. This is particularly important in situations where one may be dealing with a potentially difficult airway, as occurred in our patient, as the resulting leak of the ETT cuff and the need for tube exchange may pose a significant risk to the patient. Additionally, intraoperative exchange of ETT may be difficult or impractical if the patient is positioned non-supine or if the surgical field limits access to the tube. A good practice is to ensure that the cuff inflation system is not included in the plaster or tie as it can easily cause kinking and a rise in intracuff pressure, or it can be damaged during dressing changes. When faced with situations where tracheal reintubation or tube exchange may be difficult, salvaging the pilot balloon cuff inflating tubing to prevent cuff deflation and air leak can be valuable until the end of the procedure. These techniques can allow for reinflation of the cuff and an elective exchange of the ETT under the optimal situation.

A unique way of preventing cuff deflation when the tubing to the pilot cuff balloon is severed is to insert an intravenous cannula into the cut end of the cuff inflation tube (Figure 2). ${ }^{13}$ After the intravenous cannula is inserted into the end of the pilot tubing, the needle is removed, and a three-way stopcock is attached (Figure 3). With this connection, the ETT cuff can be re-inflated using an attached syringe, and the stopcock creates a secure seal preventing the cuff from deflating (Figure 3A). However, a potential complication of blind ETT cuff re-inflation with the hypodermic syringe, especially in the absence of the pilot balloon, which allows for a subjective estimation of the cuff pressure by palpation, is the possibility of overinflation to higher pressures with risk of severe injuries including tracheal mucosal ischemia, ulceration, necrosis, tracheoesophageal fistula or even tracheal rupture. ${ }^{14,15}$ To ensure that the reinflated cuff is not over-inflated, a cuff pressure monitor can be attached to the three-way stopcock, and intracuff pressure measured to the recommended cuff pressure of 15-25 $\mathrm{mmHg}^{16}$ (Figure 4). The size of the intravenous cannula to use depends on the size of the ETT that must be repaired. Using this temporary repair method, we tested how the various cannula sizes fit the different ETT pilot tubing to allow for cuff reinflation to provide data for reference (Table 1). Sill proposed a similar repair method but used a needle inserted into the cut end of the pilot balloon tubing. ${ }^{17} \mathrm{~A}$ major limitation to this method is that the needle can potentially cut through the cuff tubing or cause injury in an emergency setting. A final technique described by Rao et al is to insert the cut end of the pilot
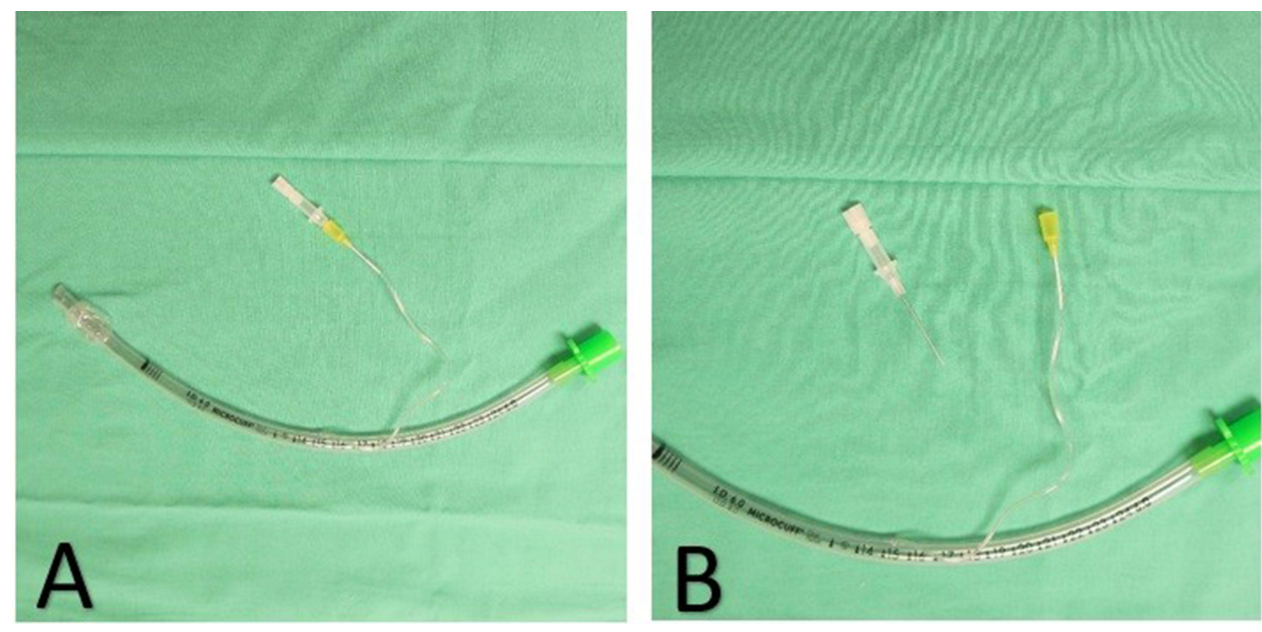

Figure 2 A 24-gauge intravenous cannula inserted into the cut end of the pilot balloon tubing of the endotracheal tube (A). A 24-gauge intravenous cannula with the needle removed connected to the cut end of the pilot balloon tubing $(\mathbf{B})$. 

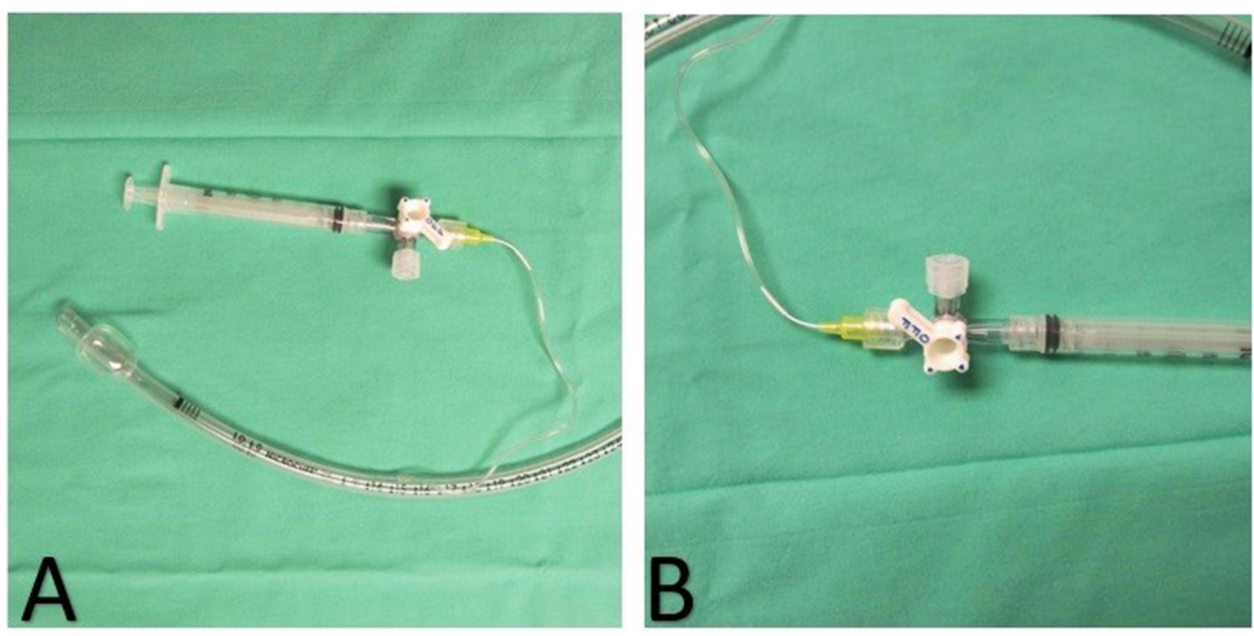

Figure 3 A 24-gauge intravenous cannula inserted into the cut end of the pilot balloon tubing of the endotracheal tube with a three-way stopcock and syringe attached to allow for cuff inflation (A). A 24-gauge intravenous cannula with the needle removed connected to the cut end of the pilot balloon tubing with a three-way stopcock and hypodermic syringe attached to prevent cuff deflation (B).

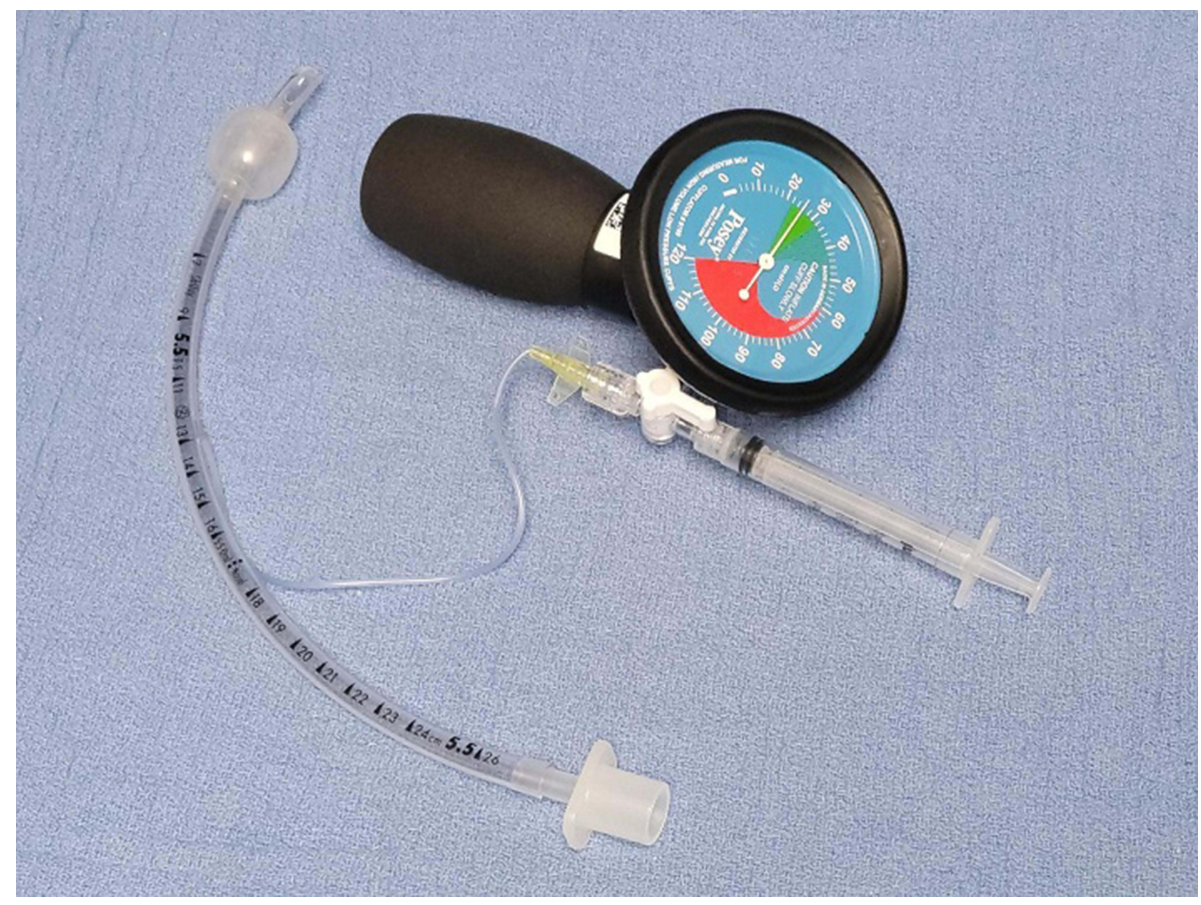

Figure 4 Intracuff pressure can be measured by connecting a cuff pressure monitor to the three-way stopcock attached to the syringe and the 24 -gauge intravenous cannula inserted into the cut end of the pilot balloon tubing of the endotracheal tube.

balloon tubing into the locking adaptor from an epidural connecter and then connecting it to a three-way stopcock. ${ }^{18}$ It should be noted that ETT cuff rupture or dysfunction can also occur independently of pilot balloon incompetency due to cuff trauma from direct laryngoscope, product defects, or changes in patient positioning. ${ }^{19}$ In the event of cuff rupture, temporary measures such as continuous insufflation of the ETT cuff with air, cuff inflation with a viscous liquid such as normal saline or lidocaine jelly have been described. ${ }^{20,21}$ However, it should be noted that such options for the management of cuff rupture may not be feasible in situations where the defect or leak is significant or during prolonged surgeries, which would mean larger volumes of viscous fluids will be needed to maintain cuff pressure.

Several temporary repair methods have been described which will allow rapid and immediate repair of a cut pilot 
Table I Cuffed Endotracheal Tube Size with Intravenous Cannula for Repair

\begin{tabular}{|l|c|}
\hline Cuffed ETT Size (mm) & IV Cannula Size (Gauge) \\
\hline $3,3.5,4,4.5$ & - \\
$5,5.5$ & 24 \\
$6,6.5,7$ & 22,24 \\
$7.5,8$ & $18,20,22$, \\
\hline
\end{tabular}

balloon tubing. These techniques allow repair while the ETT is in place. The cuff can then be re-inflated to allow for effective ventilation and later exchange of the ETT in an elective fashion. The choice of which technique or method is mainly dependent on personal preference, equipment availability, and provider comfort and experience. Given its readily available in all anesthetizing locations, we favor the use of a standard intravenous cannula. The size used will depend on the size of the ETT. Regardless of the method chosen, it is vital to ensure that the repair has an adequate seal and restoration of a normal cuff pressure to allow for proper ventilation and minimize aspiration and subglottic injury risk.

\section{Disclosure}

The authors report no conflicts of interest in this work.

\section{References}

1. Bhardwaj N. Pediatric cuffed endotracheal tubes. J Anaesthesiol Clin Pharmacol. 2013;29(1):13-18. doi:10.4103/0970-9185.105786

2. Tobias JD. Pediatric airway anatomy may not be what we thought: implications for clinical practice and the use of cuffed endotracheal tubes. Paediatr Anaesth. 2015;25(1):9-19. doi:10.1111/pan.12528

3. Weiss M, Dullenkopf A, Fischer JE, Keller C, Gerber AC; European Paediatric Endotracheal Intubation Study G. Prospective randomized controlled multi-centre trial of cuffed or uncuffed endotracheal tubes in small children. Br J Anaesth. 2009;103(6):867-873. doi:10.1093/ bja/aep290

4. Eschertzhuber S, Salgo B, Schmitz A, et al. Cuffed endotracheal tubes in children reduce sevoflurane and medical gas consumption and related costs. Acta Anaesthesiol Scand. 2010;54(7):855-858. doi:10.1111/j.1399-6576.2010.02261.x
5. Denny JT, Mungekar SS, Landgraf BR, et al. An unusual cause of failure to ventilate. $J$ Investig Med High Impact Case Rep. 2018;6:2324709618781174.

6. Kaddoum RN, Chidiac EJ, Zestos MM, Ahmed Z. Electrocauteryinduced fire during adenotonsillectomy: report of two cases. $J$ Clin Anesth. 2006;18(2):129-131. doi:10.1016/j.jclinane.2005.09.032

7. Szmuk P, Ezri T, Evron S, Roth Y, Katz J. A brief history of tracheostomy and tracheal intubation, from the Bronze Age to the Space Age. Intensive Care Med. 2008;34(2):222-228. doi:10.1007/s00134-007-0931-5

8. Dunn PF, Goulet RL. Endotracheal tubes and airway appliances. Int Anesthesiol Clin. 2000;38(3):65-94. doi:10.1097/00004311200007000-00006

9. Bayeux R. Tubage of the larynx in the croup. Medical Press. 1897;6:29-33. French.

10. Litman RS, Weissend EE, Shibata D, Westesson PL. Developmental changes of laryngeal dimensions in unparalyzed, sedated children. Anesthesiology. 2003;98(1):41-45. doi:10.1097/00000542200301000-00010

11. Dalal PG, Murray D, Messner AH, Feng A, McAllister J, Molter D. Pediatric laryngeal dimensions: an age-based analysis. Anesth Analg. 2009;108(5):1475-1479. doi:10.1213/ane.0b013e31819d1d99

12. Wani TM, Bissonnette B, Engelhardt T, et al. The pediatric airway: historical concepts, new findings, and what matters. Int J Pediatr Otorhinolaryngol. 2019;121:29-33. doi:10.1016/j.ijporl.2019.02.041

13. Kovatsis PG, Fiadjoe JE, Stricker PA. Simple, reliable replacement of pilot balloons for a variety of clinical situations. Paediatr Anaesth. 2010;20(6):490-494. doi:10.1111/j.1460-9592.2010.03313.x

14. Liu J, Zhang X, Gong W, et al. Correlations between controlled endotracheal tube cuff pressure and postprocedural complications: a multicenter study. Anesth Analg. 2010;111(5):1133-1137. doi:10.1213/ANE.0b013e3181f2ecc7

15. Fan CM, Ko PC, Tsai KC, et al. Tracheal rupture complicating emergent endotracheal intubation. Am J Emerg Med. 2004;22 (4):289-293. doi:10.1016/j.ajem.2004.04.012

16. Sole ML, Penoyer DA, Su X, et al. Assessment of endotracheal cuff pressure by continuous monitoring: a pilot study. Am J Crit Care. 2009;18(2):133-143. doi:10.4037/ajcc2009441

17. Sills J. An emergency cuff inflation technique. Respir Care. 1986;31:199-201.

18. Rao AK, Chaudhuri S, Joseph TT, Kamble D, Gotur G, Venkatesh S. Severed cuff inflation tubing of endotracheal tube: a novel way to prevent cuff deflation. Anesth Essays Res. 2014;8(2):243-246. doi:10.4103/0259-1162.134523

19. Hiller KN, Aijazi H. Endotracheal tube pilot line leak in the single use ILMA-Fastrach. Anesth Patient Saf Found. 2013;27:70-71.

20. Schubert A, Von Kaenel W, Ilyes L. A management option for leaking endotracheal tube cuffs: use of lidocaine jelly. $J$ Clin Anesth. 1991;3(1):26-31. doi:10.1016/0952-8180(91)90202-X

21. Rudlof B, Lauterbach C. Intracuff saline infusion for the short-term repair of an endotracheal tube cuff leak. Anesthesiology. 1999;90 (6):1801. doi:10.1097/00000542-199906000-00059

\section{Publish your work in this journal}

Medical Devices: Evidence and Research is an international, peerreviewed, open access journal that focuses on the evidence, technology, research, and expert opinion supporting the use and application of medical devices in the diagnosis, monitoring, treatment and management of clinical conditions and physiological processes. The identification of novel devices and optimal use of existing devices which will lead to improved clinical outcomes and more effective patient management and safety is a key feature of the journal. The manuscript management system is completely online and includes a very quick and fair peer-review system. Visit http:// www.dovepress.com/testimonials.php to read real quotes from published authors. 